\title{
The On-orbit Performance of DAMPE Trigger System
}

\author{
Yongqiang Zhang ${ }^{1,2}$, Yang Liu ${ }^{1 *}$, Jianhua Guo ${ }^{1}$ \\ ${ }^{1}$ Key Laboratory of Dark Matter and Space Astronomy, Purple Mountain Observatory, CAS, \\ Nanjing 210008, China \\ ${ }^{2}$ University of Chinese Academy of Sciences, Yuquan Road 19, Beijing 100049, China \\ E-mail: liuy@pmo.ac.cn
}

The DArk Matter Explorer (DAMPE) was launched into a sun-synchronous orbit at the altitude of $500 \mathrm{~km}$ on Dec 17, 2015. Its main scientific objective is to detect electrons and photons in the range of $5 \mathrm{GeV} \sim 10 \mathrm{TeV}$ with a high energy resolution. A first investigation to the flight data shows that the trigger system operates quite well. The trigger rate is controlled at a level of $70 \sim 100 \mathrm{~Hz}$ except in South Atlantic Anomaly region. This rate is properly high and will not cause much dead time to the detection. The trigger efficiency for high energy electrons is almost $100 \%$, which makes the detector very suitable for fulfilling its tasks.

35th International Cosmic Ray Conference - ICRC2017

10-20 July, 2017

Bexco, Busan, Korea

${ }^{*}$ Speaker. 


\section{Introduction}

The DArk Matter Particle Explorer (DAMPE) is a space science satellite which was successfully launched into a $500 \mathrm{~km}$ high solar-synchronous orbit on Dec. 17th, 2015. Its scientific goals include precisely measuring the spectra of cosmic rays electrons and Gamma rays in the range of $5 \mathrm{GeV}$ to $10 \mathrm{TeV}$ for the evidence of dark matter particles, measuring the flux of nuclei up to 500 $\mathrm{TeV}$ to study the origin and propagation of the cosmic rays [1]. DAMPE will also play an important role in finding new high energy cosmic gamma ray sources (Gamma-Ray Bursts (GRBs), pulsars et, al.).

DAMPE is consisted of four sub-detector and as shown in Fig. 1 , from the top to the bottom are : the Plastic Scintillator Detector (PSD), the Silicon Tracker (STK), The BGO Calorimeter (BGO), the neutron Detector (NUD). The PSD serves as anti-coincidence system and for charge reconstruction; the main task of STK is to reconstruct the trajectory of the incident particle; the BGO measures the deposit energy and distinguishes electrons from protons according to the topological differences of their cascade shower development; the NUD gives auxiliary information for electron-proton separation. Except for the sub-detectors described above, DAMPE has a energybased trigger system which decreases the event rate to an acceptable level for data acquisition (DAQ) system and filters out the trivial events.

In this paper we will firstly give an brief introduction to DAMPE trigger system, including its hardware design and the operation settings. Then the on-orbit performance (trigger rate and trigger efficiency for electrons) will be presented. With these results, we will illustrate the DAMPE trigger system operates in a good state and fulfil its tasks for cosmic ray detection.

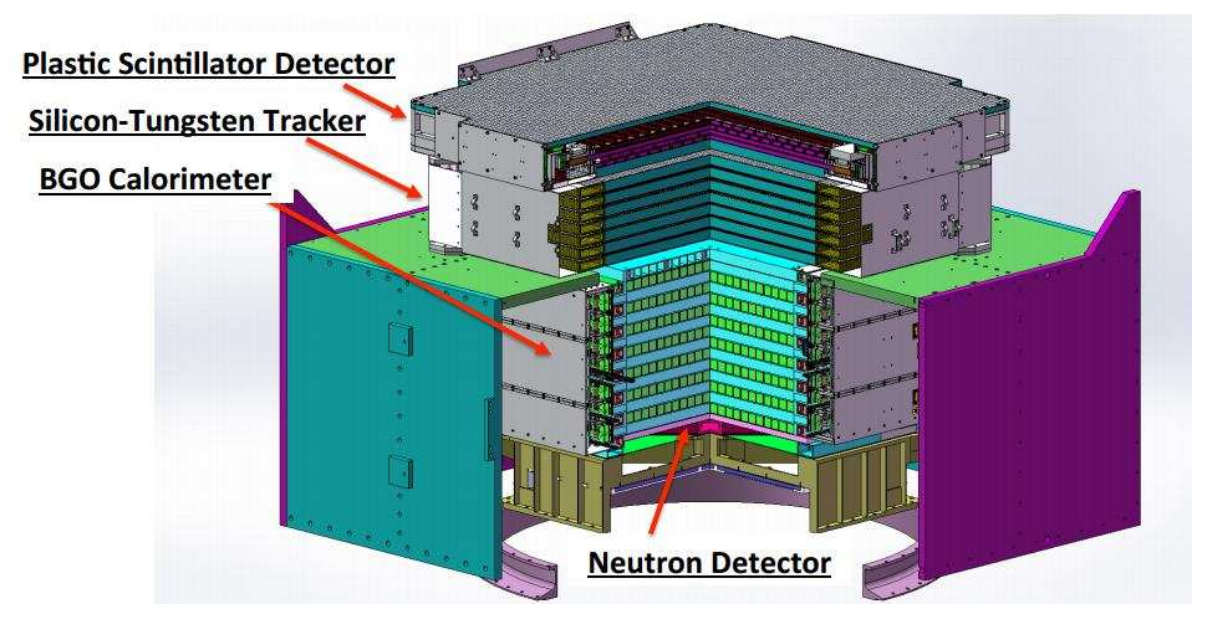

Figure 1: The Architecture of DAMPE

\section{Trigger System of DAMPE}

Like many other experiments, the trigger system of DAMPE plays a key role in selecting physics events of interest, removing the background and decreasing the size of the data set. One of the most important physics objectives of DAMPE is to measure the cosmic ray electron spectrum up to $10 \mathrm{TeV}$ and search for evidence of dark matter. Since the flux of proton is 3 orders in 
magnitude higher than that of electron, the main background for electron measurement comes from the protons. The energy deposits in the top several layers of calorimeter are significantly different for electrons and protons. We configure a high energy (HE) trigger which is sensitive to electrons and photons based on this difference. DAMPE needs the minimum ironizing particle (MIP) samples and unbiased events to calibrate the detector responses so we designed MIPs trigger and unbiased trigger to collect them. Besides, we implement a low energy (LE) trigger to select the gamma rays with the energy of several hundred MeV. We have 4 sub-triggers for scientific data taking.

The DAMPE Trigger system utilizes the information from the BGO calorimeter. As shown in Fig. 2, the calorimeter consists 14 layers of 22 BGO crystal bars with the size of $25 \mathrm{~mm} \times 25 \mathrm{~mm} \times$ $600 \mathrm{~mm}$ each. The neighbour layers are arranged with the bars oriented along orthogonal directions. The total thickness of the calorimeter is about $32 X_{0}$ or 1.6 nuclear interaction length. All these 308 crystal bars are mounted with a Hamamatsu R5610A-01 photomultiplier tubes (PMT) on both side. The light intensity is attenuated by a filter clinging to the PMT photocathode window to avoid saturation. For one side of the bars in a layer, the attenuation is greater and marked as $\mathrm{N}$ side; the other side with smaller attenuation is noted as $\mathrm{P}$ side. To achieve a large dynamic range, the signals of PMTs are lay out from the 2nd, 5th, 8th dynode. In total, there are 1848 readouts from BGO calorimeter. We use the signals from the top 4 layers and bottom 4 layers for trigger.

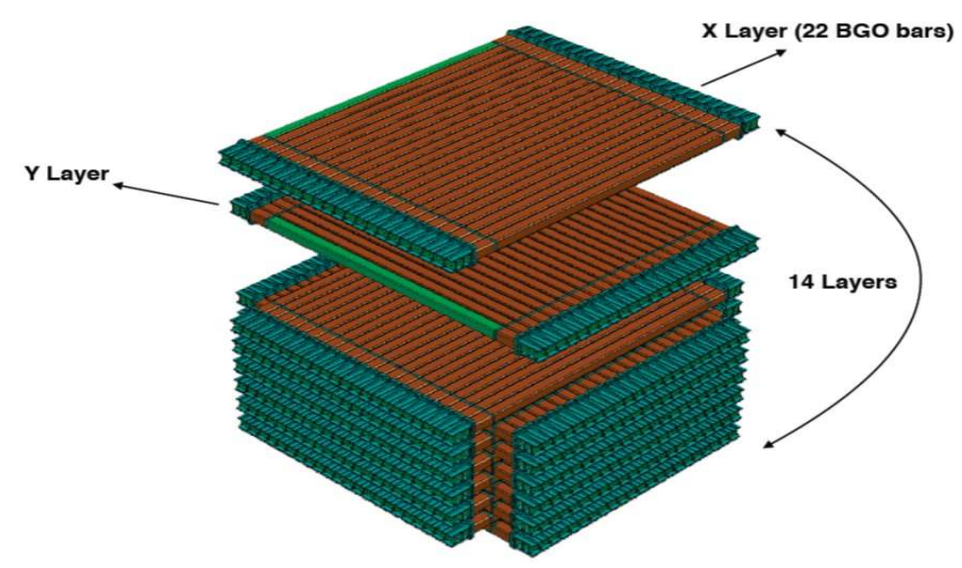

Figure 2: Schematic view of the DAMPE BGO calorimeter

The front end electronic (FEE) boards receive the current pulses from PMTs. The IDEAS VATA160 ASIC chips are the main processors doing the charge measurements and generating "hit" signal for trigger. The VA part of VATA160 has 32 analogue input channels and each channel consists of a charge-sensitive pre-amplifier (CSA), a CR-RC shaping amplifier, a sample-hold circuit $(\mathrm{SH})$; the TA part for each channel has a faster shaper and a comparator. After pre-amplified, the signals from PMT dynodes are shaped into a narrow pulse and discriminated by the comparators. One VATA160 chip will process the 22 channels of the same dynode (5 or 8), same side and same layer. All the 22 outputs of TA part are internally OR'ed together, so if at least one of these 22 channels whose signal amplitude is higher than the threshold, a "hit" signal will be yielded and sent to the trigger board. The trigger board implements the trigger decision logic with a fash based 
FPGA (Field Programmable Gate Array) chip. All the sub-trigger logic settings are shown in Tab. 1. The "L1_P_dy5" denotes the hit signal from the 1st Layer, the P side and the 5th dynode for instance. The global trigger signal is made with the four types of sub-triggers by an "Or-ed" logic operation. To keep the DAQ system work in good state, all these sub-triggers (except HE) are pre-scaled with appropriate factors. [2]

\begin{tabular}{|c|c|c|c|}
\hline Trigger Type & Logic & Energy Threshold & Pre-scale factor \\
\hline \multirow{4}{*}{$\mathrm{HE}$} & L1_P_dy5 & $\sim 10$ MIPs & \multirow{4}{*}{1} \\
\hline & \& L2_P_dy5 & $\sim 10 \mathrm{MIPs}$ & \\
\hline & \& L3_P_dy5 & $\sim 10 \mathrm{MIPs}$ & \\
\hline & \& L4_N_dy8 & $\sim 2$ MIPs & \\
\hline \multirow{3}{*}{ MIPs (Type I) } & L3_P_dy8 & $\sim 0.4$ MIPs & 4 (low latitude $\left( \pm 20^{\circ}\right)$ ) \\
\hline & \& L11_P_dy8 & $\sim 0.4$ MIPs & \\
\hline & \& L13_P_dy8 & $\sim 0.4$ MIPs & Turn Off (other region) \\
\hline \multirow{3}{*}{ MIPs (Type II) } & L4_P_dy8 & $\sim 0.4$ MIPs & 4 (low latitude $\left.\left( \pm 20^{\circ}\right)\right)$ \\
\hline & \& L12_P_dy8 & $\sim 0.4$ MIPs & \\
\hline & \& L14_P_dy8 & $\sim 0.4$ MIPs & Turn Off (other region) \\
\hline \multirow{4}{*}{ LE } & L1_N_dy8 & $\sim 0.4$ MIPs & \multirow{4}{*}{8 (low latitude $\left( \pm 20^{\circ}\right)$ ) } \\
\hline & \& L2_N_dy8 & $\sim 0.4$ MIPs & \\
\hline & \& L3_N_dy8 & $\sim 2$ MIPs & \\
\hline & \& L4_N_dy8 & $\sim 2$ MIPs & \\
\hline \multirow{2}{*}{ Unbiased } & (L1_P_dy8 \& L1_N_dy8) & $\sim 0.4 \mathrm{MIPs} \sim 0.4 \mathrm{MIPs}$ & 512 (low latitude $\left( \pm 20^{\circ}\right)$ ) \\
\hline & | (L2_P_dy8 \& L2_N_dy8) & $\sim 0.4$ MIPs $\sim 0.4$ MIPs & 2048 (other region) \\
\hline
\end{tabular}

Table 1: The sub-trigger settings of DAMPE

\section{Trigger Rate}

The trigger rate of DAMPE varies with the fluxes of the cosmic ray particles on the orbit. The magnetic field of the earth has a blocking effect on the charged particles and correspondingly the fluxes are changed. In low latitude area, the geomagnetic cutoff is high and the incident particles with low energy can not reach the near-earth space. They may drift along the magnetic lines to the pole region where the cutoff is lower, then these particles could reach the low altitude space. Due to this effect the charged particle fluxes in the pole region are higher than those around the equator. An other thing should be mentioned here is the South Atlantic Anomaly (SAA) where the intensity of the magnetic field is only about half of other areas with the same latitude. The particle fluxes in SAA are higher and therefore the trigger rate will sharply jump up when flying into SAA. Fig. 3 shows how the trigger rate varies in a period of 3 orbits. We use different colors to mark the trigger rates of 4 sub-triggers. The trigger rates will suddenly jump up (down) when the pre-scale factor are changed, which is more obvious in unbiased trigger and LE trigger. We can see the largest contribution of trigger rate is from HE trigger. The HE trigger rate will be about $70 \mathrm{~Hz}$ in the pole region and in the equator region this value will decrease to $20 \mathrm{~Hz}$. The unbiased trigger rate is constrained in several $\mathrm{Hz}$ level and the LE trigger is in the middle. The MIPs trigger only functions 
in low latitude $\left( \pm 20^{\circ}\right)$. In SAA, the unbiased trigger rate could reach its peak value of $120 \mathrm{~Hz}$. Except for SAA, the total trigger rate is less than $100 \mathrm{~Hz}$.

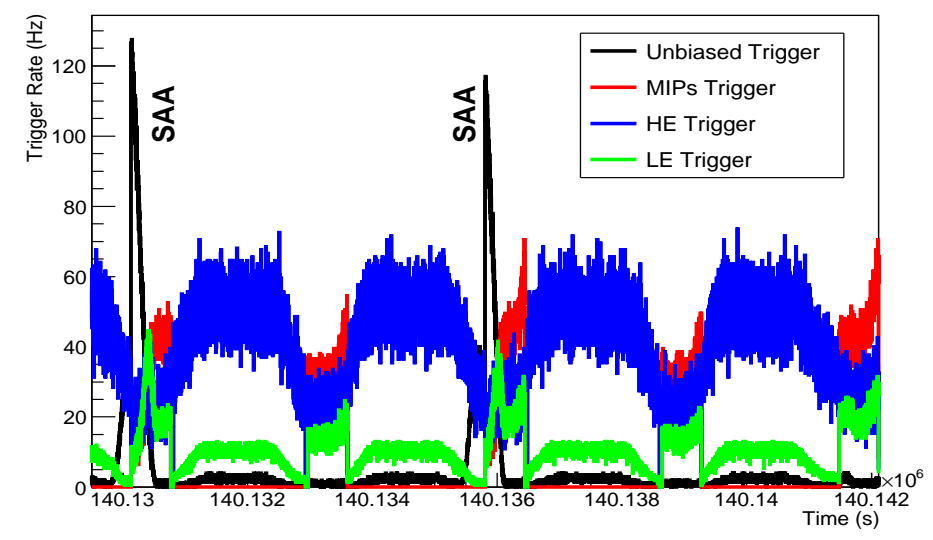

Figure 3: The trigger rate of DAMPE in 3 consecutive orbits

\section{Trigger Efficiency}

It is very important to secure DAMPE has an almost $100 \%$ trigger efficiency for high energy electrons and photons. For electron spectrum measurement, we rely on the HE trigger since it has a basic electron-proton separation power and no pre-scale effect. As described before, the HE trigger is configured based on the features of electro-magnetic cascade shower of electrons. We can evaluate the HE trigger efficiency for electrons in two ways. One is by Monte Carlo and the other is data-driven methods. These two methods could be used for a crosscheck. For Monte Carlo, we first develop a method to calibrate the trigger thresholds then put these parameters in GEANT4 simulation. The MC programme will precisely tell us whether an event passes the trigger criteria or not. In data driven way, We select a pure electron sample. From this sample we get the number of events pass both the HE trigger and the unbiased trigger, also we can get the number of events pass unbiased trigger (No HE trigger requirement here). The ratio of these two numbers is taken as the HE trigger efficiency. Fig. 4 shows the result of such a comparison. The trigger efficiency for electrons goes up with energy increasing. The efficiency changes dramatically in the low energy region, then the curve becomes flat. The results from MC and data match quite well.

\section{5. summary}

The trigger criteria of DAMPE is based on the energy deposit in the BGO calorimeter. Four sets of sub-triggers is configured according to the different physics motivations. The trigger rate is adjusted by setting proper pre-scale factors in different orbit circumstances. Since the launch in Dec. 2015, the trigger system has operated in a stable state for 18 months. On-flight performance illustrates the trigger system is effective and robust.

The trigger efficiency for electrons is estimated using MC and data-driven methods. They give consistent result. The efficiency will go up to almost $100 \%$ in high energy range, which has 


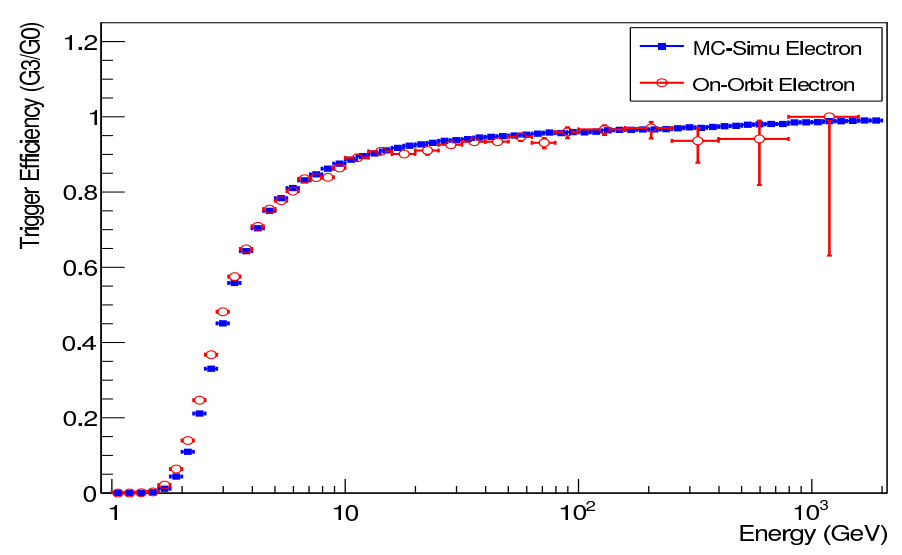

Figure 4: High energy trigger efficiency on electrons

a special meaning in electron spectrum measurement. It also can be taken as an evidence that the trigger system is operating well as we expect.

\section{Acknowledgments}

This work is partly supported by the National Key R\&D Program of China, Grant No. 2016YFA0400201 and the Joint Research Fund in Astronomy under cooperative agreement between the National Natural Science Foundation of China (NSFC) and CAS, Grant No. U1531126.

\section{References}

[1] CHANG Jin. Dark Matter Particle Explorer: The First Chinese Cosmic Ray and Hard $\gamma$-ray Detector in Space. Chin. J. Space Sci., 2014, 34(5): 550-557

[2] GUO Jianhua, ZHANG Yunlong, FENG Changqing. The Trigger System of DAMPE. The 33rd International Cosmic Ray Conference, Rio de Janeiro. 2013. 